\title{
EINSTEIN EQUATIONS VIA NULL SURFACES
}

\author{
S. IYer, E.T. NEWMAN \\ Dept. of Physics and Astronomy, University of Pittsburgh, Pittsburgh, PA 15213, USA \\ AND C. Kozameh \\ FaMaf, University of Cordoba, Cordoba, Argentina
}

\section{Dedicated to Prof. Iwo Bialynicki-Birula on the occasion of his 60th birthday}

We present a version of the vacuum Einstein equations where the field equations are defined for cross-sections of a line bundle over the sphere and where the manifold of solutions is four-dimensional and defines the space-time itself. The cross-sections themselves become the characteristic surfaces of the space-time.

PACS numbers: 04.20.-q

\section{Introduction}

We will present here a brief discussion of the progress we have made on a program that has occupied us for close to fifteen years. As a lengthy detailud exposition of this work is being prepared, we will only give the results in broad outline.

The basic idea of the program is the reformulation of General Relativity in terms of concepts and variables that are to be treated or considered, from our point of view, as more fundamental than space-time itself; i.e., where space-time arises as a derived concept from a "primary structure" and a metric, satisfying the Einstein equations, appears automatically but as a secondary idea.

Though shortly we will give physical meaning to the ideas, we begin with our fundamental mathematical structure, a line bundle over the (metric) two-sphere, coordinatized with the (complex) stereographic coordinates $(\zeta, \bar{\zeta})$ on the $S^{2}$ component and $u$ as the bundle coordinate. The central idea is that we are able to give a specific set of (integro-differential) equations, symbolically given by

$$
\mathcal{D}(u, \zeta, \bar{\zeta}, \text { data })=0
$$

(which unfortunately are quite involved), whose solutions $u=Z(\zeta, \bar{\zeta})$ define cross-sections of the bundle - with the cross-sections having the following set of properties:

1. There are a four-parameter family of solutions to $\mathcal{D}(u, \zeta, \bar{\zeta}$, data $)=0$; each solution thus has the form $u=Z\left(x^{a}, \zeta, \bar{\zeta}\right)$, with $x^{a}$ as the four parameters. The solution space itself is to be interpreted as a space-time manifold $\mathcal{M}$. 
2. The solutions, which are two-dimensional surfaces in three dimensions, will be "global" in the sense that the only type of singularities associated with the surfaces are "swallow-tail" singularities.

3. The solutions $u=Z\left(x^{a}, \zeta, \bar{\zeta}\right)$, for fixed $(u, \zeta, \bar{\zeta})$, can be interpreted as a three-parameter set of characteristic surfaces of some conformal metric on $\mathcal{M}$.

4. The conformal metric can be explicitly calculated from the cross-sections $u=Z\left(x^{a}, \zeta, \bar{\zeta}\right)$.

5. There will be a choice of conformal factor (obtained from the solution of a second order linear o.d.e.) so that the associated metric satisfies the asymptotically-flat vacuum Einstein equations. By varying the "data" in $\mathcal{D}(u, \zeta, \bar{\zeta}$, data $)=0$, all asymptotically flat metrics can be "found" in this manner.

6 . Though the equations are quite complicated and there is little hope that they can be solved exactly, there is a simple straightforward perturbation procedure (starting with Minkowski space) which is (or at least appears to be) unambiguous at each step.

\section{Geometric interpretation}

The structures we have just described have a simple interpretation in terms of an asymptotically flat space-time and in fact have been synthesized (as we will try to demonstrate) from the asymptotically-flat vacuum solutions of the Einstein equations.

We assume a certain familiarity $[1,2]$ with the basic ideas of the theory of asymptotically flat space-times and just review a few notational points. An asymptotically flat space-time comes "endowed" with a (future) conformal boundary $\mathcal{J}^{+}$ (future null infinity - the endpoints of future directed null geodesics), which has the structure of a light-cone, $S^{2} \times R . \mathcal{J}^{+}$is identified with the line bundle over $S^{2}$ of the previous section; again coordinatized by $(\zeta, \bar{\zeta}, u)$. If we now consider an arbitrary space-time point (with local coordinates $x^{a}$ ), the intersection of the light-cone from $x^{a}$ with $\mathcal{J}^{+}$is interpreted as the cross-section $u=Z\left(\zeta, \bar{\zeta}, x^{a}\right)$, i.e.,

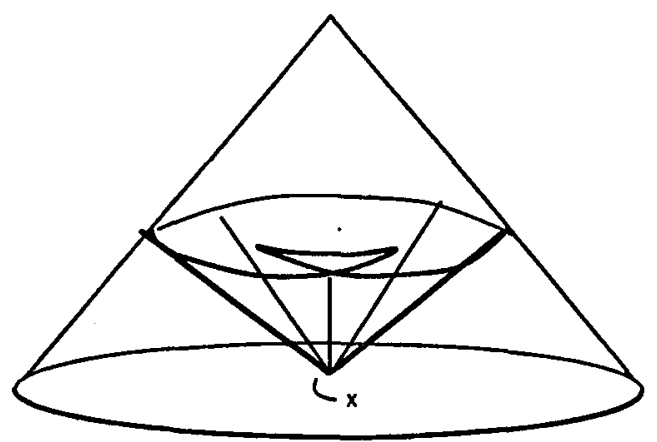

Fig. 1. The intersection of light-cone from $x$ with null infinity - with swallow-tail singularity. 
property 1 (see Fig. 1). The data referred to in $\mathcal{D}(u, \zeta, \bar{\zeta}$, data), is the characteristic data for an asymptotically flat space-times, i.e., the Bondi news function. Its singularity structure. (swallow-tails), property 2 , follows from the theory of singularities of wave fronts [3]. The cross-sections $u=Z\left(\zeta, \bar{\zeta}, x^{a}\right)$ have an alternative interpretation; not only are they the intersections of the future light-cones from $x^{a}$ with $\mathcal{J}^{+}$, they are also the past light-cones from the (fixed) point $(\zeta, \bar{\zeta}, u)$ on $\mathcal{J}^{+}$, i.e., they are families (parametrized by $(\zeta, \bar{\zeta}, u)$ ) of characteristic surfaces; property 3 . Now; by fixing $x^{a}$ and varying $\zeta, \bar{\zeta}$ one can reconstruct the conformal metric at $x^{a}$; property 4 . If we had started with a space-time conformal to a vacuum Einstein space (i.e., with $\mathcal{D}(u, \zeta, \bar{\zeta}$, data) $=0$ ) then there exists a second order linear o.d.e. for the determination of the appropriate conformal factor; property 5 . A perturbation scheme, property 6 , based on systematically correcting the characteristic surfaces (starting with Minkowski space), exists and appears to be easily implementable.

\section{Derivation}

Unfortunately the derivation of the set $\mathcal{D}(u, \zeta, \bar{\zeta}$, data $)=0$ is not simple; it requires considerable technical detail and, for completeness, becomes quite involved. We will thus restrict ourselves to giving simply an outline of the basic ideas and leave the details to both earlier publications [4-7] and forthcoming publications.

a) We begin with an asymptotically-flat vacuum space-time $\mathcal{M}$ and introduce (as we mentioned earlier) the set of characteristic surfaces through each point $\boldsymbol{x}^{a}$, i.e., $u=Z\left(x^{a}, \zeta, \bar{\zeta}\right)$, defined either as the past light-cones from the point $(u, \zeta, \bar{\zeta})$ of $\mathcal{J}^{+}$or the intersection of the future light-cone from $x^{a}$ with $\mathcal{J}^{+}$. From $Z\left(x^{a}, \zeta, \bar{\zeta}\right)$ we will obtain certain "intrinsic" coordinates (intrinsic in the sense that they are constructed directly from the characteristic surfaces themselves) which will then be used to describe "intrinsically" the conformal geometry of the original space-time. The idea is that the characteristic surfaces themselves become the basic "variables".

b) We then describe how the conformal metric can be reconstructed from these characteristic surfaces in terms of the intrinsic coordinates. Two important things happen here; (1) the metric, up to a conformal factor, is explicitly expressible in terms of the intrinsic coordinates and (2) there are auxiliary conditions placed on the "characteristic surfaces" that imply that the surfaces are the characteristic surfaces of some conformal metric.

c) Finally, we will show how to impose "field equations", i.e., the conformal Einstein equations, on the characteristic surfaces and then how to break the conformal invariance and obtain the Einstein equations themselves.

a) The intrinsic coordincles

From the characteristic surfaces, i.e., from

$u=Z\left(x^{a}, \zeta, \bar{\zeta}\right)$,

by applying the operators eth and eth-bar [1] (essentially just the $\zeta, \bar{\zeta}$ derivatives), we can define the four scalar functions

$$
\theta^{i}=\theta^{i}\left(x^{a}, \zeta, \bar{\zeta}\right)=\left(\theta^{0}, \theta^{+}, \theta^{-}, \theta^{1}\right)=(u, \omega, \bar{\omega}, R)
$$


by

$$
\begin{array}{ll}
u=Z\left(x^{a}, \zeta, \bar{\zeta}\right), & R=\partial \bar{\partial} Z\left(x^{a}, \zeta, \bar{\zeta}\right), \\
\omega=\partial Z\left(x^{a}, \zeta, \bar{\zeta}\right), & \bar{\omega}=\bar{\partial} Z\left(x^{a}, \zeta, \bar{\zeta}\right),
\end{array}
$$

which, with the inverse transformation

$$
x^{a}=x^{a}\left(\theta^{i}, \zeta, \bar{\zeta}\right)
$$

can be thought of as an $S^{2}$ 's worth of coordinate transformations between $x^{a}$ and the "intrinsic coordinates" $\theta^{i}$, which are parametrized by $(\zeta, \bar{\zeta})$.

The next idea is to "construct"

$$
\begin{aligned}
& \partial^{2} Z \equiv \lambda\left(x^{a}, \zeta, \bar{\zeta}\right)=\Lambda\left(\theta^{i}, \zeta, \bar{\zeta}\right), \\
& \bar{\partial}^{2} Z \equiv \bar{\lambda}\left(x^{a}, \zeta, \bar{\zeta}\right)=\bar{\Lambda}\left(\theta^{i}, \zeta, \bar{\zeta}\right),
\end{aligned}
$$

where (5) has been used to eliminate $x^{a}$ in terms of $\theta^{i}$. The $\Lambda\left(\theta^{i}, \zeta, \bar{\zeta}\right)$, its conjugate and its gradients are to be very important variables for us. Note that they are expressed in terms of the intrinsic coordinates.

We stress the essential idea that if $\Lambda\left(\theta^{i}, \zeta, \bar{\zeta}\right)$ and its conjugate are given and (6) is considered as an equation for $Z$, then it is possible to show that there is a four-parameter family of solutions, $u=Z\left(x^{a}, \zeta, \bar{\zeta}\right)$ to $(6)$.

The plan is to code into $\Lambda\left(\theta^{i}, \zeta, \bar{\zeta}\right)$ and its conjugate the information about (1) a conformal metric and (2) a conformal metric satisfying the conformal Einstein equations. The characteristic surfaces are then to be obtained from (6).

b) The conformal metric and the "auxiliary conditions"

From the scalars $\theta^{i}$, a gradient basis $\theta_{, a}^{i}$ and the dual vectors $\theta_{i}^{a}$ can be formed; one can also express the gradient of $A$ by

$$
\Lambda_{, a}=\Lambda_{, i} \theta_{, a}^{i}=\Lambda_{0} Z_{, a}+\Lambda_{1} \partial \bar{\partial} Z_{, a}+\Lambda_{+} \partial Z_{, a}+\Lambda_{-} \bar{\partial} Z_{, a} .
$$

Since $\mathcal{M}$ has a metric, $g^{a b}\left(x^{a}\right)$, we can transform it to the intrinsic coordinates $\theta^{i}$ by

$$
g^{i j}\left(\theta^{i}, \zeta, \bar{\zeta}\right)=g^{a b}\left(x^{a}\right) \theta_{, a}^{i} \theta_{, b}^{j}
$$

so that, for example,

$$
g^{00}=g^{a b}\left(x^{a}\right) Z_{, a} Z_{, b}, \quad g^{0+}=g^{a b}\left(x^{a}\right) Z_{, a} \partial Z_{, b}, \quad g^{01}=g^{a b}\left(x^{a}\right) Z_{, a} \partial \bar{\partial} Z_{, b} .
$$

One has immediately, from the assumption that $Z$ is a characteristic surface, that

$$
g^{00} \equiv g^{a b}\left(x^{a}\right) Z_{, a} Z_{, b}=0
$$

and by taking eth and eth-bar derivatives (and with the fact that $g^{a b}$ depends only on $x^{a}$ ) that

$$
g^{0+}=0, \quad g^{0-}=0, \quad g^{01}+g^{+-}=0 .
$$

It is now possible, by repeated eth and eth-bar operations on $g^{00}=0$ to express all the components of $g^{i j}$ (except $g^{01}$ ) in terms of $\Lambda_{, i}$, i.e., one obtains [4-7] explicitly a conformal metric, with $g^{01}\left(\theta^{i}, \zeta, \bar{\zeta}\right)$ as the conformal factor (see Fig. 2)

$$
g^{i j}=g^{01} h^{i j}\left(\Lambda_{, i}\right) \text {. }
$$

In addition, one obtains two complex differential relations (independent of $g^{i j}$ ), between $\Lambda_{, i}$, that are referred to as the "auxiliary conditions"; they are conditions 


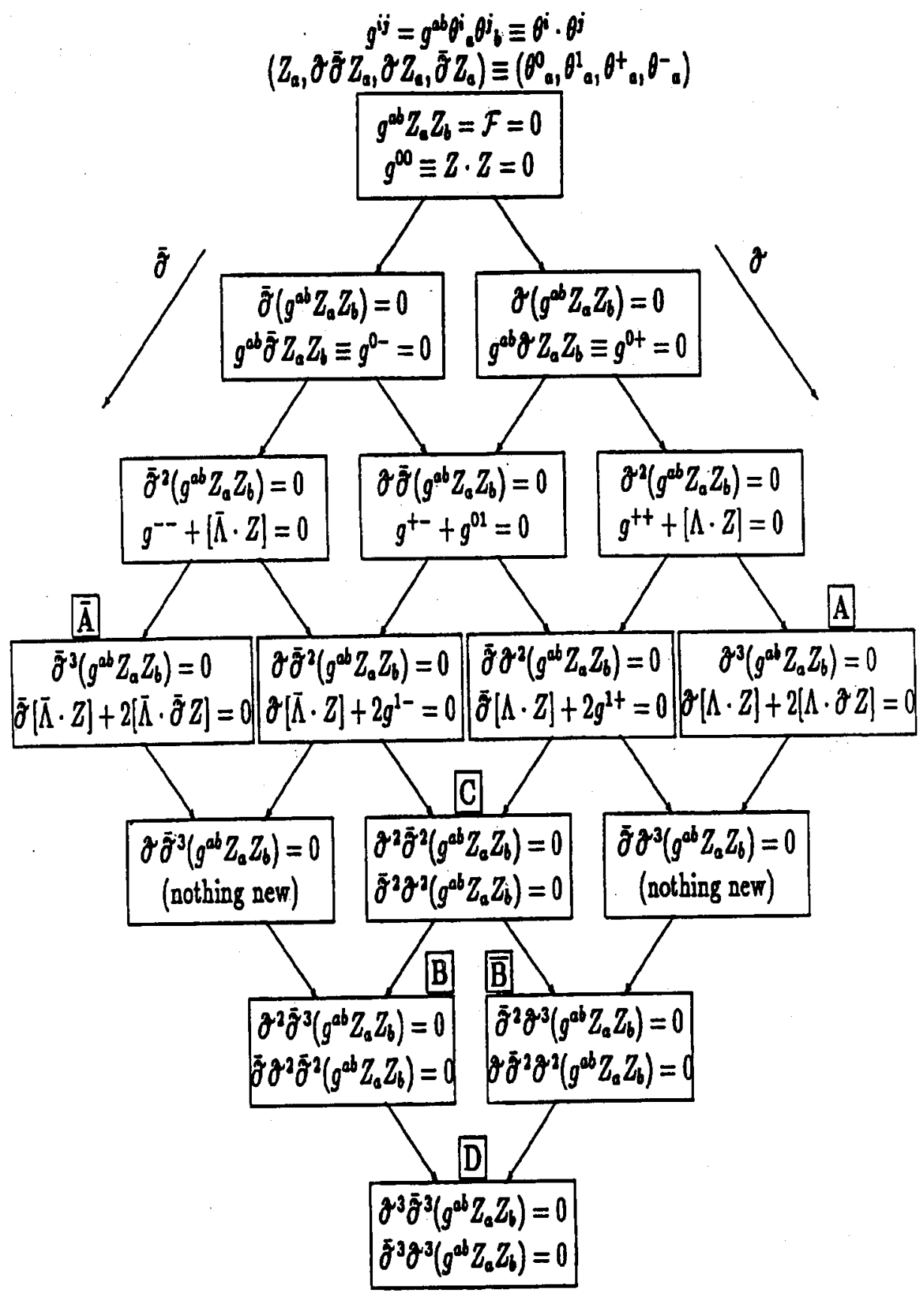

Fig. 2. Metric diamond. 
to be imposed on $\Lambda_{, i}$ so that $g^{i j}$ do indeed come from a metric $g^{a b}\left(x^{a}\right)$ via Eq. (8). We denote them by $\mathcal{A}\left(\Lambda_{, i}\right)=0$ and $\mathcal{B}\left(\Lambda_{, i}\right)=0$.

In addition to the auxiliary conditions, one finds (by direct calculation from its definition; $\left.g^{01}=g^{a b}\left(x^{a}\right) Z_{, a} \partial \bar{\partial} Z_{, b}\right)$, that though $:^{01}$ is not completely determined, a condition on its eth (and eth-bar) derivative can be obtained, namely

$$
\partial g^{01}=W\left(\Lambda_{, i}\right) g^{01},
$$

with $W(\Lambda, i)$ explicitly given. This says that $g^{01}$ is uniquely determined up to a factor depending only on $x^{a}$; i.e., its $\zeta, \bar{\zeta}$ behavior is completely determined by $\Lambda_{, i}$. Equation (11) plays an extremely important role for us.

c) The conformal Einstein equations imposed on $A$

The conformal Einstein equations can be imposed in the following fashion.

Since in our construction $Z_{, a}\left(\zeta, \bar{\zeta}, x^{a}\right)$ is a null one-form at $x^{a}$ and for all $\zeta, \bar{\zeta}$ spans the null cone, the Einstein equations, $R^{a b}=0$ (assuming the cosmological constant is zero), can be written as

$$
R^{a b} Z_{, a} Z_{, b}=0 .
$$

If we now substitute the metric from Eq. (10) into Eq. (12) we obtain a single relationship between $g^{01}$ and $\Lambda$ which has the remarkably simple form [5]

$$
D^{2} U=Q U
$$

where $D=\partial / \partial \theta^{1}=\partial / \partial R, U=\sqrt{\left(g^{01}\right)}$ and $Q=Q(\Lambda, i)$ is a relatively simple function of $\Lambda_{, i}$. Equation (13) is a linear second order o.d.e. for $\sqrt{\left(g^{01}\right)}$ given in terms of $\Lambda$ and has often been referred to as the "Einstein bundle equation". We can thus think of $g^{01}$ as a functional of $\Lambda$, i.e.,

$$
g^{01}=g^{01}(\Lambda)
$$

The boundary conditions for Eq. (13) are that $g^{01}=1$ as $R$ tends to infinity.

Now by substituting (14) into (11) we obtain a (complicated) functional equation

$$
\mathcal{L}\left(\Lambda_{, i}\right)=0
$$

for the determination of $\Lambda$. This equation, with the two auxiliary conditions, $\mathcal{A}$ and $\mathcal{B}$ and Eqs. (6), are, in essence, the conformal Einstein equation.

After some manipulation of (15), (6) and the auxiliary conditions, $\mathcal{A}\left(\Lambda_{, i}\right)=$ $\mathcal{B}(\Lambda, i)=0$, we have our set

$$
\mathcal{D}(u, \zeta, \bar{\zeta}, \text { data })=0 .
$$

The data has entered the system by integrating one of the auxiliary conditions and imposing asymptotic flatness on the "function" of integration. In reality, there is some ambiguity as what we consider is the best or simplest form for the set $\mathcal{D}(u, \zeta, \bar{\zeta}$, data $)=0$.

Once $A$ is solved for, i.e., is known, then by simply substituting it into (14), we obtain $g^{01}$, the proper conformal factor for yielding the asymptotically-flat vacuum metrics. 


\section{Examples}

\subsection{Minkowski space}

For Minkowski space Eq. (16) becomes

$\partial^{2} Z=0, \quad \bar{\partial}^{2} Z=0$.

The solution, $Z=Z_{\mathrm{M}}\left(x^{a}, \zeta, \bar{\zeta}\right)$ immediately leads, via the above construction, to Minkowski space.

\subsection{Self-dual metrics}

Many years ago we studied $[8,9]$ an unusual and remarkably simple formula"I of the self-dual vacuum Einstein equations that turns out to be a special case of the present construction, Eq. (16). With the choice of self-dual data, Eq. (16) simply becomes (with certain regularity conditions)

$$
\partial^{2} Z=\sigma_{\mathrm{B}}(Z, \zeta, \bar{\zeta})
$$

with $\sigma_{\mathrm{B}}(Z, \zeta, \bar{\zeta})$ an arbitrary spin-weight-2 function, as the data. From the point of view of the differential equation, the four $x^{a}$ are constants of integration, i.e., the solution space of the differential equation is itself the manifold in question. Directly, from these four-parameter solutions, i.e., from knowledge of the $Z\left(x^{a}, \zeta, \bar{\zeta}\right)$, the self-dual vacuum metric can be constructed.

\subsection{Linearized gravity}

For linear theory Eq. (16) takes the form

$$
\bar{\partial}^{2} \partial^{2} Z=\partial^{2} \bar{\sigma}_{\mathrm{B}}+\bar{\partial}^{2} \sigma_{\mathrm{B}}
$$

with $\bar{\sigma}_{\mathrm{B}}(u, \zeta, \bar{\zeta})$ and $\sigma_{\mathrm{B}}(u, \zeta, \bar{\zeta})$ as free characteristic data given on $\mathcal{J}^{+}$and with $u$ replaced by the Minkowski space solution for $Z_{M}$, i.e., from the solution to (17).

\section{Summary and conclusion}

Summarizing, we want to emphasize that there is something rather striking about Eq. (16); it is a set of equations for the determination of a function $Z=Z(\zeta, \bar{\zeta})$, on a line bundle over $S^{2}$. The solution space is a four-dimensional manifold with local coordinates $x^{a}$ (which are the constants of integration) that automatically comes with (from the solutions $Z\left(x^{a}, \zeta, \bar{\zeta}\right)$ ) a conformal metric. This conformal metric can be easily converted to a metric which automatically satisfies the vacuum Einstein equations. The different asymptotically-flat vacuum solutions are coded into the different choices of characteristic data, used in (16).

Without trying to make too much of it, we want to emphasize how different this formulation is from the usual one. First of all it takes characteristic surfaces as its basic variable - the details are different but the spirit is similar to Penrose's idea that points of twistor space are to be the basic entities from which space-times will be derived. The equations are very non-local - there does not appear any 
evolution in the conventional sense - the manifold $\mathcal{M}$ and the characteristic surfaces simply appear as solutions of Eq. (16). One then calculates, by simply differential and algebraic operations, the associated metric which is automatically Lorentzian and vacuum. It appears to us highly unlikely that other field theories of physical interest can be formulated in a like manner; it is this feature (among others) of classical general relativity that makes us question the meaning or even the desirability of constructing a (conventional) quantum theory of gravity.

\section{Acknowledgments}

We thank L. Mason for many enlightening and stimulating discussions. This research was supported in part by the National Science Foundation under grants No. PHY 89-04035 and PHY 92-05109. C.K. thanks CONICET for support. E.T.N. thanks NATO for support from a collaboration research grant.

\section{References}

[1] E.T. Newman, K.P. Tod, in: General Relativity and Gravitation, Ed. A. Held, Vol. 2, Plenum, New York 1980.

[2] R. Geroch, in: Asymptotic Structure of Space-Time, Eds. P. Esposito, L. Witten, Plenum Press, New York 1977.

[3] V.I. Arnold, Mathematical Theory of Classical Mechanics, Springer-Verlag, New York 1978.

[4] C.N. Kozameh, E.T. Newman, in: Topological Properties and Global Structure of Space-Time, Eds. P. Bergmann, V. de Sabbata, Plenum Press, 1986.

[5] C.N. Kozameh, E.T. Newman, in: Asymptotic Behavior of Mass and Space-Time Geometry, Ed. F.J. Flaherty, Lecture Notes in Physics, Vol. 202, Springer-Verlag, New York 1984.

[6] C. Kozameh, W. Lamberti, E.T. Newman, Ann. Phys. 206, 193 (1991).

[7] S. Iyer, C. Kozameh, E.T. Newman, J. Geom. Phys. 8, 195 (1992).

[8] R.O. Hansen, E.T. Newman, R. Penrose, K.P. Tod, Proc. R. Soc. A 363, 445 (1978).

[9] M. Ko, M. Ludvigsen, E.T. Newman, K.P. Tod, Phys. Rep. 71, 53 (1981). 